\title{
Emotion Recognition Test Functional Test
}

National Cancer Institute

\section{Source}

National Cancer Institute. Emotion Recognition Test Functional Test. NCI Thesaurus.

Code C150909.

A standardized method for measuring visibly different facial movements that was developed by Ekman et al in 1976. 